\title{
CD34 + cells in maternal placental blood are mainly fetal in origin and express endothelial markers
}

\author{
Olivier Parant ${ }^{1,2}$, Gil Dubernard ${ }^{1,2}$, Jean-Claude Challier ${ }^{1,2}$, Michèle Oster ${ }^{1,2}$, Serge Uzan ${ }^{1,2}$, Sélim Aractingi ${ }^{1,2}$ \\ and Kiarash Khosrotehrani ${ }^{1,2}$
}

Fetal CD34 + cells enter the maternal circulation during pregnancy and may persist for decades. These cells are usually depicted as hematopoietic stem/progenitor cells. Our objective was to further determine the phenotype of fetal chimeric CD34 + cells in placental maternal blood from the intervillous space (IVS). Human healthy term placentas were analyzed $(n=9)$. All fetuses were male. CD34 + cells were identified in the IVS and further characterized as fetal or maternal using $X$ and $Y$ chromosome fluorescence in situ hybridization. The phenotype of fetal cells was further analyzed using anti-CD117 (c-kit), anti-CD133, anti-CD31, anti-von Willebrand factor (vWF), anti-vimentin, anti-CD45 and anticytokeratin (CK) antibodies. We used preeclamptic placentas of male $(n=3)$ and healthy placentas of female fetuses $(n=3)$ as controls. As expected fetal cells were easily identified in the IVS and significantly increased in cases of preeclampsia. Most CD34 + cells in the IVS were of fetal origin (90\%) and were not surrounded by CK staining further showing that they were not in fetal trophoblastic villi. Similarly, about $40 \%$ of CD31 + and $6 \%$ of vimentin + cells in the IVS were fetal in origin. No CD117 + or CD133 + fetal cells were found in the IVS of examined placentas. Besides, all the CD34 + cells identified in the IVS were co-labeled with vWF or CD31, suggesting their endothelial origin. These results suggest that most CD34 + cells in maternal placental blood at term are fetal in origin from endothelial and not hematopoietic lineages.

Laboratory Investigation (2009) 89, 915-923; doi:10.1038/labinvest.2009.55; published online 1 June 2009

KEYWORDS: CD34 + cells; endothelial progenitors; FISH; microchimerism; placenta

During all human pregnancies, fetal cells enter the maternal circulation. ${ }^{1,2}$ This phenomenon starts early, as soon as at 6 weeks of gestation in a small proportion of women. ${ }^{1}$ The number of fetal cells has been estimated about $1-6$ per ml of maternal blood during the second trimester ${ }^{2}$ and seems influenced by obstetrical events such as preeclampsia, ${ }^{3}$ abortion, ${ }^{4,5}$ amniocentesis or fetal abnormalities, such as Down syndrome, ${ }^{6}$ each of which result in higher amounts of transferred cells. The phenotype of the fetal cells transferred to the mothers has also been investigated despite their low frequency. Mothers acquire a variety of cells such as nucleated erythrocytes, trophoblasts, leukocytes and even fetal progenitors. ${ }^{7}$ After gestation, fetal cells persist in a large proportion of women's circulation and tissues for decades. ${ }^{8}$ In recent years, the transfer of a variety of progenitor cells of fetal origin during gestation has been demonstrated. These cells have been shown to home to maternal injured tissues where they can adopt a variety of phenotypes depending on the host organ. ${ }^{9}$ In human studies as well as murine models, fetal cells were found to express markers of epithelia in thyroid ${ }^{10}$ tubular epithelium in kidneys, ${ }^{11}$ hepatocytes in liver ${ }^{11-13}$ and even neuronal and glial cells in the central nervous system. ${ }^{14}$ These findings suggest that progenitors with multipotent capacities have been transferred during gestation. ${ }^{15}$ However, the precise nature of these progenitors remains unknown. In many studies on maternal blood and bone marrow, investigators have described potential candidates responsible for the observed plasticity of fetal microchimeric cells. O'Donoghue $e a^{16}$ have reported the presence of fetal-derived mesenchymal stem cells in maternal blood during pregnancy and in maternal bone marrow decades after delivery. Using hematopoietic progenitor cell (HPC) culture together with PCR amplification of a Y chromosomespecific sequence, Osada et $a l^{17}$ demonstrated the presence of fetal HPCs in maternal blood immediately after gestation. Fetal CD34 + cells have been found in maternal

\footnotetext{
${ }^{1}$ UPMC Univ Paris 6, Laboratoire de Physiopathologie du Développement, EA 4053, Paris, France and ${ }^{2}$ INSERM UMR_S893, Saint-Antoine Research Center, Paris, France Correspondence: Dr K Khosrotehrani, MD, PhD, Faculté de médecine Pierre et Marie Curie: Saint-Antoine, Laboratoire de Physiopathologie du développement, UPRES EA4053, UPMC Univ Paris 6, 27 rue de Chaligny, Paris 75012, France. E-mail: kiarash.khosrotehrani@tnn.aphp.fr 
blood during gestation. ${ }^{18}$ In addition, persistence of fetal $\mathrm{CD} 34+\mathrm{CD} 38+$ and CD34+CD38 - cells has been described up to 27 years after delivery. ${ }^{8}$ Similar observations of fetal CD34 + cells have also been reported in maternal blood from primates and in maternal cytapheresis products after granulocyte colony-stimulating factor mobilization. ${ }^{19,20}$ Accordingly, lymphopoiesis of fetal origin has been described in maternal thymus during and after gestation, suggesting the relevance and functionality of these presumed fetal HPCs. ${ }^{21}$ The expression of the CD34 cell-surface antigen is associated with an increased frequency of hematopoietic stem cell (HSC)/HPC. However, it is not a specific marker. Other studies have described the presence of fetal-derived blood vessels in maternal inflammatory skin and tumors, suggesting the transfer of fetal endothelial progenitors. ${ }^{22,23}$ The CD34 antigen is also expressed on endothelial progenitors and many of the endothelial fetal cells in the above-mentioned studies were labeled with CD34. ${ }^{23}$ Because numerous studies have described the placenta as a niche for HSCs expressing CD34 and CD117 (c-kit), ${ }^{24,25}$ we hypothesized that the placental blood circulating in the intervillous space (IVS) was a privileged site for the detection of the transfer of CD34+ cells from the fetus to the mother. We thus conducted a study on maternal blood from the IVS to further determine the phenotype of fetal chimeric CD34 + cells in maternal blood.

\section{MATERIALS AND METHODS}

\section{Placentas}

After institutional review board approval and informed consent, we obtained placentas from healthy women undergoing a term delivery: vaginal delivery $(n=4)$ or cesarean section $(n=5)$. All women delivered a male fetus. Immediately after collection, placentas were sectioned transversally, fixed in formalin without additional washing procedures and embedded into paraffin. This procedure allows minimizing contamination of maternal areas of the placenta with fetal blood. Paraffin inclusion was performed with the maternal side of the placenta facing the surface of the bloc. Serial sections $(5 \mu \mathrm{m})$ were obtained on superfrost + slides from each sample. We also obtained control specimen from preeclamptic women delivering a male fetus $(n=3)$ and healthy women delivering a female fetus $(n=3)$. Preeclampsia was defined by high blood pressure (diastolic and systolic blood pressure of at least 90 or $140 \mathrm{mmHg}$, respectively) with proteinuria (presence of $300 \mathrm{mg}$ or more of protein in a 24-h urine collection) after 20 weeks of gestational age according to the American College of Obstetricians and Gynecologists definition. The patients included presented severe preeclampsia with evidence of ischemic lesions on placental examination.

\section{Fluorescence In Situ Hybridization}

Fluorescence in situ hybridization (FISH) was performed as previously described. ${ }^{26}$ We used $\mathrm{X}$ and $\mathrm{Y}$ chromosome probes labeled respectively with $\mathrm{Cy} 3$ (red) and fluorescein isothiocyanate (FITC) (green) mapping to Xp11.1q11.1, a satellite centromeric region of the $\mathrm{X}$ chromosome, and Yq12, satellite III region of the $\mathrm{Y}$ chromosome (Vysis, Downers Grove, IL, USA).

\section{Combined FISH with Immunostaining}

We combined FISH and immunostaining as previously described for frequent antigens such as cytokeratin (CK) or CD45. ${ }^{9,27}$ The procedure was however modified for less frequent antigens. Vimentin, CD34, CD117 (c-kit), CD133, von Willebrand stainings were first performed using standard immunofluorescence. After detection of cells of interest and proper localization using coordinates, slides were submitted to $\mathrm{X}$ and $\mathrm{Y}$ chromosome FISH. Cells were then retrieved and assessed as male or female. Immunofluorescence was performed as follows: after deparaffinization, rehydration and antigen retrieval procedures, sections were blocked with $20 \%$ goat serum and incubated with primary antibodies for $1 \mathrm{~h}$. After several washes, sections were incubated with a secondary antibody. After scoring, slides were processed for in situ hybridization to establish the fetal or maternal origin of the cells of interest. In addition, positive slides $(n=17)$ were labeled with anti-CK antibody after FISH. Cells of interest were retrieved as described and assessed as $\mathrm{CK}+$ or $\mathrm{CK}-$ to differentiate chimeric fetal cells $(\mathrm{CK}-)$ and sloughed fetal trophoblast $(\mathrm{CK}+)$.

\section{Antibodies}

The antibodies used were monoclonal mouse anti-CD34 (clone Qbend10, dilution 1:40), mouse anti-vimentin (clone LN6, dilution 1:100; Sigma, www.sigmaaldrich.com), monoclonal mouse anti-CD133 (dilution 1:50; Miltenyi Biotech, www.miltenyibiotec.com/), polyclonal rabbit anti-CD31 (PECAM-1) (dilution 1:50; Santa Cruz Biotechnology Inc., www.scbt.com), monoclonal mouse antiCD31 (dilution 1:20), polyclonal rabbit anti-CD117 (c-kit, dilution 1:20) (Dako Corporation, Carpinteria, CA, USA), monoclonal mouse anti-CK (clone AE1/AE3; Dako Corporation), monoclonal mouse anti-CD45 (clones 2B11 + PD7/27; Dako Corporation) and polyclonal rabbit antivon Willebrand antigen (dilution 1:400; Abcam, Cambridge, UK). We used a goat anti-mouse antibody labeled with Texas Red and a goat anti-rabbit antibody labeled with Fluorescein as secondary antibodies (Jackson ImmunoResearch, West Grove, PA, USA). All slides were counterstained with 4',6diamidino-2-phenylindole (DAPI) observed under a fluorescence microscope (Leica, Deerfield, IL, USA) with a QImaging digital camera (Media Cybernetics, Silver Spring, MD, USA).

\section{Confocal Laser Scanning Microscopy}

We used confocal laser scanning microscopy to demonstrate the colocalization of cell-surface markers in the fetal cells isolated in the IVS. Confocal microscope analysis was carried out using the TCS SP2 Leica (Lasertechnik GmbH), equipped 

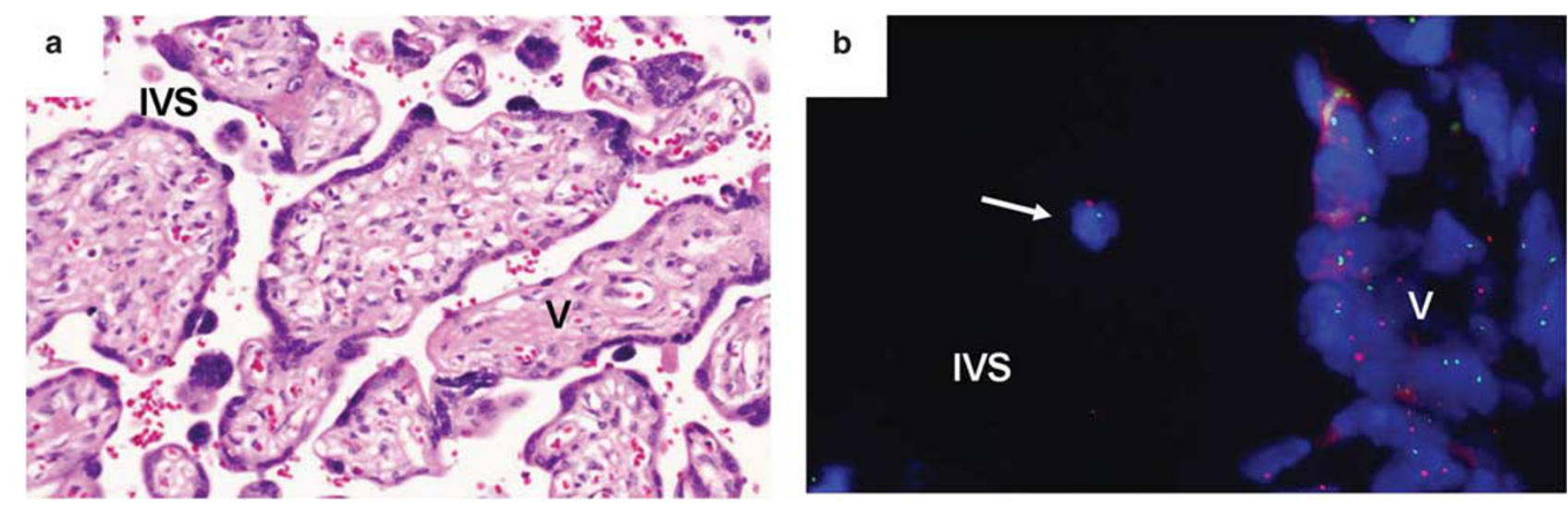

Figure 1 Presence of fetal cells in the intervillous space. (a) Photomicrograph depicting villi (V) and intervillous space (IVS) in a section of normal term placenta after hematoxylin-eosin staining $(\times 100)$. (b) Identification of a fetal cell in the IVS, harboring an X (in red) and a Y (in green) chromosome after FISH $(\times 400)$. Of note, the cell is distant from surrounding villi. Nuclei are labeled with DAPI (blue).

with a $\times 40$ objective. For each optical section, doublefluorescence images were acquired. A focal series of sections was collected for each specimen. The focus step between the sections was generally $1-1.5 \mu \mathrm{m}$. Selected paired sections were then processed to produce single composite overlay images (color-merged).

\section{Scoring and Statistical Analysis}

On each section, we identified the IVS and the villi, respectively, as maternal and fetal areas. The IVS is filled with maternal placental blood. In this area, male fetal cells were identified as having one $\mathrm{X}$ (red) and one $\mathrm{Y}$ (green) chromosome. Maternal cells were identified as having two $\mathrm{X}$ chromosomes. Sections were scored if more than $70 \%$ of the nuclei had at least one FISH signal. To conclusively identify a cell, the following criteria were requested: (1) presence of two red signals (female) or one red and one green signal (male) corresponding to the size and shape of adjacent $\mathrm{X}$ or $\mathrm{Y}$ chromosomes inside a blue-colored nucleus, (2) presence of a minimal distance between the two signals corresponding to at least the size of one of them and (3) presence of intact and recognizable nuclear borders. Cells that had zero or only one identifiable $\mathrm{X}$ or $\mathrm{Y}$ chromosome were considered as 'undetermined'. Within each section, we estimated the total number of nucleated cells (NCs) in the IVS by counting three areas of approximately ten $\times 400$ power fields on each section as previously described. ${ }^{9,28}$ For each case, the number of microchimeric cells found was reported to the total number of NCs examined in the IVS. CD45, CD34, CD31, vimentin, CD133 and CD117 cells were identified in the IVS and counted if they were isolated and distant from adjacent villi. Labeled cells found next to damaged villi were excluded. The number of identified labeled cells was reported to the total number of NCs examined in the IVS. For each individual cell stained with any of the antibodies, spatial location on the slide was recorded using coordinates. FISH was performed on the same slides, cells were retrieved and their fetal or maternal origin analyzed.

We compared the average number of fetal CD34 + cells and CD31 + cells using Student's $t$-test for continuous and $\chi^{2}$-test for binary variables. A $P$-value of less than 0.05 was considered significant.

\section{RESULTS}

\section{Fetal Cells can be Easily Identified in the IVS}

The IVS is the maternal side of the placenta and contains maternal blood in close contact to fetal trophoblastic villi. To maximize the observation of fetal cells entering maternal blood, we assessed their presence in the IVS where fetal and maternal tissues are the closest (Figure 1a). Besides, we also assessed preeclamptic placentas, known to have increased numbers of fetal chimeric cells, as a positive control to validate our methods. We obtained serial sections from term placentas of male fetuses. An average of 6-7 (range 2-15) slides were processed for each sample respectively from healthy and preeclamptic term placentas. We did not observe any differences between placentas obtained by cesarean section or vaginal delivery. On each section, villi were easily recognizable and delimited the IVS. We first performed FISH using $\mathrm{X}$ and $\mathrm{Y}$ chromosome probes to sort male fetal cells from their maternal counterparts in the IVS. The surrounding villi consisted entirely of XY + cells confirming that the fetuses were male. In all placentas, fetal cells harboring an $\mathrm{X}$ and a $\mathrm{Y}$ chromosome could be easily identified in the IVS (Figure 1b). Placentas from female fetuses $(n=3)$ never displayed any XY + cells. Among the male fetal cells in the IVS, 26\% expressed CK suggesting their trophoblastic origin. These cells could be part of trophoblastic villi as well as trophoblast cells detached from villi in the maternal placental blood. The remaining CK- cells were therefore within the IVS and not connected to the villi. In healthy term 

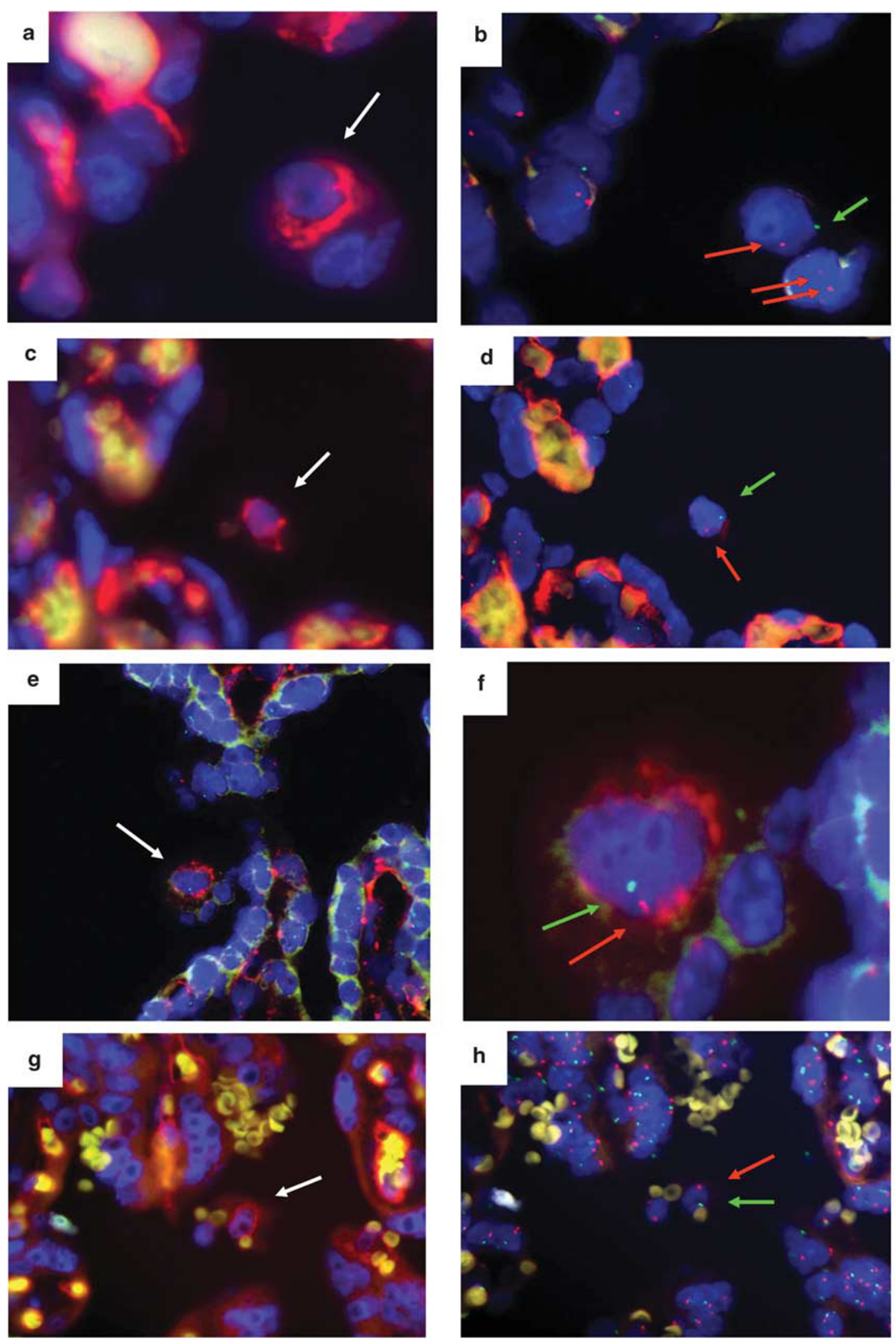
placentas, out of 125 NCs from the IVS that could be correctly assessed, 50 were identified as fetal (40\%). As expected, the average frequency of fetal cells was significantly higher in preeclamptic placentas $(58 \%, 172 / 299)(P=0.001)$.

\section{Most CD34 + Cells in the IVS are of Fetal Origin}

We next assessed the expression of CD34. Fetal blood vessels in the villi were stained in all specimens and were considered as an internal quality control of the staining. Individual cells stained with anti-CD34 antibody could be found in the IVS from healthy and preeclamptic placentas (Figure 2). These cells were significantly more frequent in preeclamptic compared to healthy term placentas $(15.6 \pm 3.3 v s 3.8 \pm 1.3 / 10000$ total NCs, $P<0.001$ ) (Table 1). We next determined on each slide whether the CD34 + cells were fetal or maternal in origin. After FISH, $70 \%$ of the CD34+ cells could be successfully relocated based on their coordinates on the slide. Among these, $75 \%$ had an appropriate FISH signal allowing them to be clearly identified as fetal or maternal (Figure 2 and Supplementary Figure 1). We identified $90 \%$ of the $\mathrm{CD} 34+$ cells as fetal in origin in the IVS from healthy placentas ( $89 \%$ in preeclamptic placentas) as witnessed by the presence of one $\mathrm{X}$ and one $\mathrm{Y}$ chromosome signals (Table 1). Therefore, the expression of CD34 seems to be very specific and almost restricted to fetal cells in the IVS. In addition, to clearly eliminate the possibility that isolated fetal CD34+ cells in the IVS could be trophoblasts or represent a section of a fetal vessel within trophoblastic villi, we performed triple staining using CD34, CK and $\mathrm{X}$ and $\mathrm{Y}$ chromosome FISH sequentially (Figure 3). Fetal CD34 + cells did not express $\mathrm{CK}$ and were not surrounded by CK expressing cells.

\section{Characterization of the IVS Fetal CD34 + Cells}

To further characterize the IVS CD34 + cells, we used various antibodies to sort their hematopoietic or endothelial phenotype (Table 2). We first used CD117 (c-kit) as a marker of HPCs. CD117-expressing cells could be found in some villi or in the basal plate (Supplementary Figure 2). However, CD117 + cells could never be identified in the IVS despite high numbers of examined cells both in normal and preeclamptic placentas (43 146 examined NCs from 12 slides and 42471 examined NCs from 9 slides, respectively). Similarly, CD133, another marker of early progenitors of endothelial or hematopoietic origin, did never stain any cell within the IVS although rare cells within the villi were stained. The absence of these markers prompted us to evaluate the capacity of the CD34 + cells from the IVS to express endothelial markers.

CD31 staining, used as a marker of endothelial cells, also revealed the presence of $49 \pm 17.7$ stained per 10000 NCs in the IVS. CD31 + cells within the IVS were male in lower proportions when compared to CD34 (30\% of evaluated cases compared to $90 \%$, respectively, $P=0.001$ ) (Figure $2 \mathrm{~g}$ and h). Similarly, we evaluated vimentin expression in the IVS, as a marker of mesenchymal cells (Supplementary Figure $3)$. Of note, the antibody clone used here did not stain macrophages but only fibroblasts, myofibroblasts and endothelial cells. The frequency of vimentin-expressing cells in the IVS was higher than CD $34+(66.2 \pm 20.3$ vs $3.8 \pm 1.3$ per 10000 NCs in the IVS). However, a much lower proportion of vimentin-expressing cells proved to be fetal in origin (6 vs 90\%, $P<0.001)$. Interestingly and despite these large differences, the number of vimentin + cells and CD34 + cells reported to 10000 NCs in the IVS that were indeed fetal was quite similar, suggesting that fetal CD34+

Table 1 Quantification of CD34+ cells of fetal or maternal origin in the intervillous space

\begin{tabular}{|c|c|c|c|c|c|c|c|}
\hline & \multirow[t]{2}{*}{ NCs in the IVS (n) } & \multirow[t]{2}{*}{ Slides ( $n$ ) } & \multirow[t]{2}{*}{ CD34 cells ( $n$ ) } & \multirow[t]{2}{*}{ Ratio CD34/10 ${ }^{4} \mathrm{NCs}$ ( \pm s.e.) } & \multicolumn{3}{|c|}{$\mathrm{FISH}^{\mathrm{a}}$} \\
\hline & & & & & $X Y$ & $X X$ & Undet. \\
\hline Normal placenta third trimester $(n=9)$ & 162252 & 57 & 62 & $3.8 \pm 1.3^{b}$ & $29(90 \%)$ & 3 & 11 \\
\hline Preeclampsia third trimester $(n=3)$ & 53984 & 11 & 84 & $15.6 \pm 3.3^{b}$ & $62(89 \%)$ & 8 & 7 \\
\hline
\end{tabular}

FISH: fluorescence in situ hybridization; IVS: intervillous space; NCs: nucleated cells.

${ }^{\mathrm{a}}$ Number of cells harboring $\mathrm{X}$ and $\mathrm{Y}(\mathrm{XY})$, two $\mathrm{X}(\mathrm{XX})$ or undetermined (undet.) for $\mathrm{X}$ or $\mathrm{Y}$ chromosomes after FISH.

${ }^{\mathrm{b}} P<0.001$.

Figure 2 Detection of fetal chimeric CD34 + or CD31 + cells in the intervillous space (IVS). Term placentas were scanned for the presence of CD34 + or CD31 + cells in the IVS. (a and c) Individual cells (arrow) in the IVS expressing CD34 (Texas Red: red). Nuclei are labeled with DAPI (blue; $\times 400)$. Retrieved cells were next assessed using $X$ and $Y$ chromosome FISH. (b and d) Same cells than (a) or (c) after FISH $(\times 400)$. Red and green arrows indicate $X$ and $Y$ chromosomes, respectively. (b) Photomicrograph showing a CD34 + fetal cell (harboring X and $Y$ chromosome) and a CD34-maternal cell (harboring two $X$ chromosomes). (e and f) CD34 + microchimeric cell (arrow) after combining FISH and CD34 immunolabeling $(\times 400$ and $\times 1000$ magnification, respectively). (g and $\mathbf{h}$ ) Individual cell (arrow) in the IVS expressing CD31 (Texas Red: red; $\times 400)$. (h) Same cell than $(\mathbf{g})$ after FISH $(\times 400)$. Red and green arrows indicate $\mathrm{X}$ and $\mathrm{Y}$ chromosomes, respectively, attesting the cell's fetal origin. 

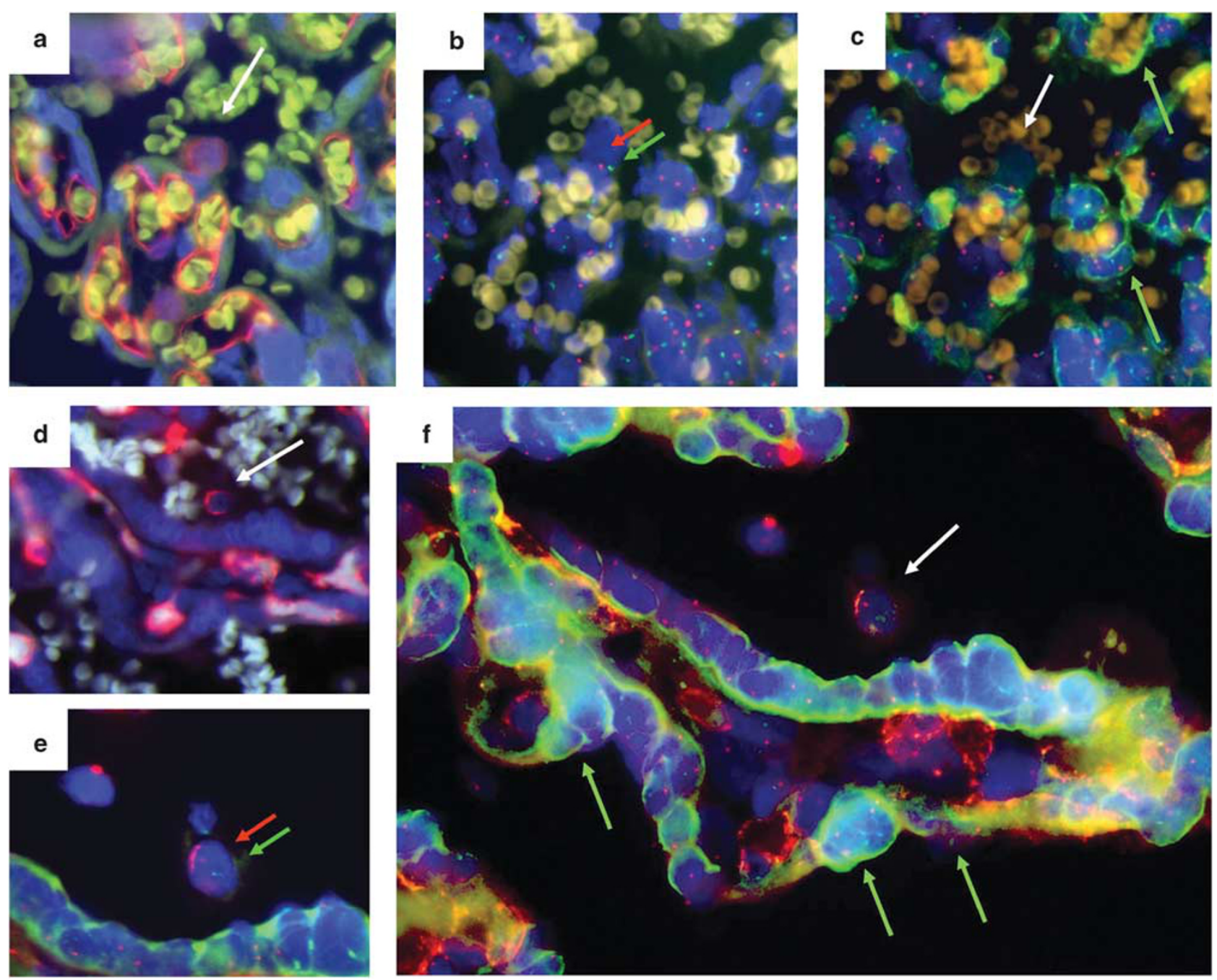

Figure 3 Isolated fetal CD34 + cells in the IVS do not express cytokeratin (CK-). (a and d) Individual cells (white arrows) in the IVS were stained with CD34 (Texas Red: red). DAPI was used as a nuclear counterstain (in blue). Autofluorescent red blood cells appear yellow or white. (b and e) Same cells than (a and d) after FISH ( $\times$ 400). Red and green arrows indicate $X$ and $Y$ chromosomes, respectively, demonstrating that both cells are fetal in origin. (c and $\mathbf{f}$ ) Same cells than (a and $\mathbf{d}$ ) after cytokeratin staining (FITC: green; $\times 400$ ). Isolated fetal cells are CK - (white arrows). Green arrows show CK labeling of villous trophoblasts surrounding the fetal CD34 + CK- cells in the IVS.

may also express vimentin (Table 2). Finally, using similar procedures to label CD45 + cells, we show that a very small proportion of the CD45 + cells in the IVS are fetal in origin $(1 \%)$ as shown in Supplementary Figure 4.

We next double-stained CD34 + cells in the IVS of term placentas with anti-von Willebrand factor (vWF) or antiCD31 using confocal microscopy (Figure 4). In these experiments, all CD34 + cells from the IVS were also labeled with CD31. Of note some CD31 + cells in the IVS were not co-labeled with CD34 as expected. vWF is a protein from endothelial cells that is not commonly expressed on hematopoietic progenitors even during fetal life. ${ }^{29}$ The blood vessels inside the villi were stained with $\mathrm{CD} 34$ as well as von Willebrand with a characteristic granular staining that was considered as an internal positive control on each slide.
CD34 + cells in the IVS were in proportions comparable to our initial experiments with anti-CD34 single labeling. All the CD34 + cells identified in the IVS were labeled with vWF and harbored the characteristic granules (Figure 4). After FISH, we could also show that CD34 + vWF + cells from the IVS were fetal in origin (Figure 4b).

\section{DISCUSSION}

Maternal spiral arteries in the decidua are directly connected to the IVS. The maternal blood from the IVS is collected in uterine veins and then in the peripheral circulation. As a result, the number of fetal cells in the IVS should be at its maximal level before dilution or immune clearance in the general circulation. ${ }^{30,31}$ Therefore, our study represents an 

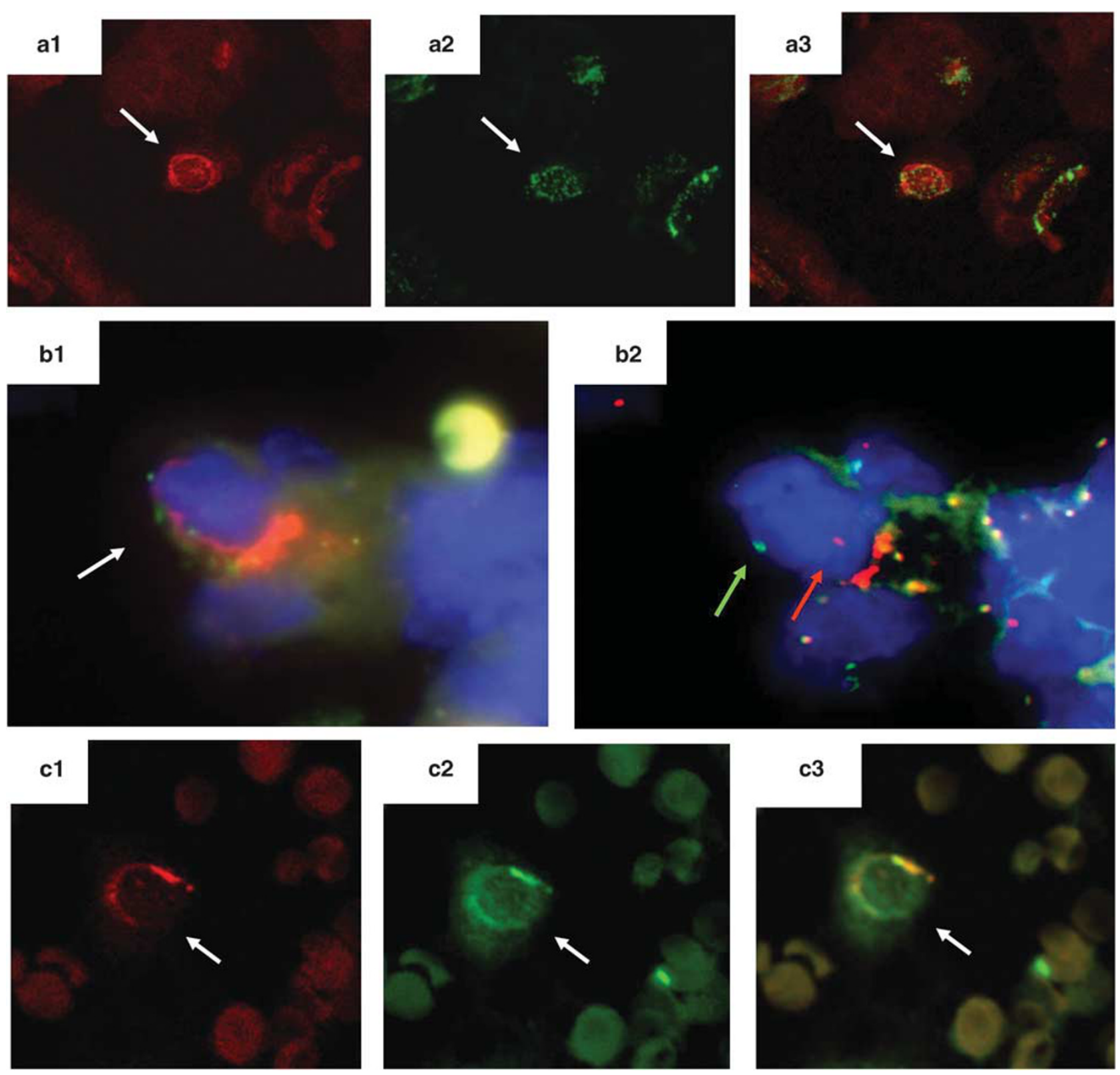

Figure 4 Fetal CD34 + cells express von Willebrand factor or CD31. Photomicrographs showing CD34 labeling (Texas Red: red) combined with von Willebrand factor (FITC: green) (a and b) or CD31 (FITC: green) (c) staining. (a) Confocal laser microscopy images of double-stained CD34/vWF isolated cell ( $\times 400 / 1 \mu \mathrm{m}$ focus step). CD34 (red) (a1), von Willebrand factor (green) (a2) and composite overlay image (a3) (white arrow). Typical granular pattern of green stain depicting von Willebrand factor expression $(\mathbf{a 2}, \mathbf{b} 1)$ can be shown on a CD34 + (red) isolated cell in the IVS. (b2) represents the same cell shown in (b1) after FISH: the CD34 + von Willebrand + cell harbors an X (red arrow) and a Y (green arrow) chromosome showing its fetal origin ( $\times 1000)$. (c) Confocal laser microscopy images of double-stained CD34 (red)/CD31 (green) isolated cell ( $\times$ 400). CD34 (red) (c1), CD31 (green) (c2) and composite overlay image (c3) (white arrow).

interesting approach for the evaluation of fetal cell microchimerism in particular in determining the phenotype of rare populations. In this study, we showed that fetal cells represented up to $40 \%$ of the total NCs in the IVS from normal term placentas. This high proportion of fetal cells allowed us to more easily investigate their phenotype. We showed that $\mathrm{CD} 34+$ cells in the IVS were almost exclusively fetal in origin. They did not express CK, excluding the possibility that they might be trophoblasts. We did not find any fetal cell expressing c-kit or CD133 in the IVS, suggesting that the term $\mathrm{CD} 34+$ cells were not hematopoietic progenitors. However, CD31 + fetal cells as well as vimentin + fetal cells could be retrieved. Finally, the fetal $\mathrm{CD} 34+$ cells also expressed CD31 or vWF. This strongly suggests that the fetal $\mathrm{CD} 34+$ cells transferred to the mother at term were in majority endothelial cells rather than HPCs.

For the purpose of our study, placentas were collected immediately after delivery and quickly fixed in formalin. This 
Table 2 Distribution of fetal cells in different populations of maternal placental blood in the intervillous space

\begin{tabular}{|c|c|c|c|c|c|c|c|}
\hline \multirow[t]{2}{*}{ Marker } & \multirow[t]{2}{*}{ NCs in the IVS (n) } & \multirow[t]{2}{*}{ Slides (n) } & \multirow[t]{2}{*}{ Stained cells ( $n$ ) } & \multirow{2}{*}{$\begin{array}{l}\text { Ratio stained cells } / 10^{4} \mathrm{NCs} \\
( \pm \text { s.e. })\end{array}$} & \multicolumn{3}{|c|}{$\mathrm{FISH}^{\mathrm{a}}$} \\
\hline & & & & & $X Y$ & $X X$ & Undet. \\
\hline CD34 & 162252 & 57 & 62 & $3.8 \pm 1.3$ & $29(90 \%)$ & 3 & 11 \\
\hline CD31 & 44788 & 10 & 22 & $49 \pm 17.7$ & $3(30 \%)$ & 7 & 4 \\
\hline Vimentin & 24459 & 7 & 162 & $66.2 \pm 20.3$ & $6(6 \%)$ & 100 & 37 \\
\hline CD45 & ND & 5 & 134 & ND & $2(1 \%)$ & 88 & 2 \\
\hline
\end{tabular}

FISH: fluorescence in situ hybridization; IVS: intervillous space; NCs: nucleated cells; ND: not performed.

${ }^{a}$ Number of cells harboring $X$ and $Y(X Y)$, two $X(X X)$ or undetermined (undet.) for $X$ or $Y$ chromosomes after FISH.

procedure minimizes the chances of contamination of the IVS by fetal blood or cells. Such procedure cannot be performed on first trimester placentas or trophoblast tissue due to the low density of trophoblastic villi and the high risk for degradation of the IVS by washing and fixation steps. Previous studies have demonstrated an increased level of fetal cell microchimerism in the circulation of women with preeclampsia. ${ }^{3}$ In accordance, our results showed a higher frequency of fetal cells in the IVS from preeclamptic placentas. These data strengthen the validity of our approach suggesting that IVS in situ analysis is representative of maternal placental blood. In our study, human placentas were cut in transversal sections. This allowed the clear identification of the trophoblastic villi and the IVS. Recently, Vernochet $e a^{32}$ did a close analysis in mouse placenta that showed EGFP + fetal cells in the maternal part of the placenta. Altogether, we strongly believe that the study model used here represents an unbiased powerful tool to analyse fetomaternal trafficking.

$\mathrm{CD} 34+$ cells may have various functions. Osada et $a^{17}$ used long-term cultures of hematopoietic progenitors from the peripheral blood of previously pregnant women. By PCR targeting the SRY gene, they found that $1.70 \%$ of HPCs in the maternal blood were indeed of fetal origin. Recently, the placenta has been described as a source of hematopoietic progenitors with colony-forming and repopulation abilities. The placental hematopoietic progenitors have been described as CD34 + c-kit + cells. ${ }^{24}$ Our study confirmed the frequent presence of fetal CD34 + cells in the IVS. Interestingly, no fetal cell expressed c-kit or CD133, suggesting that the majority of fetal CD34+ cells were not hematopoietic progenitors. Nevertheless, the absence of c-kit + fetal cells in term IVS does not exclude the transfer of fetal HPCs at a lower frequency or at earlier stages during gestation. Indeed, in their study, Bianchi et $a l^{8}$ described the presence of $\mathrm{CD} 34+\mathrm{CD} 38+$ cells of fetal origin that are undoubtedly hematopoietic in origin. Similarly, we have shown the transfer of fetal lymphoid progenitors during gestation that can form mature lymphocytes in maternal thymus and bone marrow. ${ }^{21}$ Here, CD34 + fetal cells appeared clearly to express vWF, CD31 and possibly vimentin. The association of such markers is very evocative of an endothelial rather than a hematopoietic phenotype. Our data suggest therefore that most fetal CD34 + transferred cells are not hematopoietic progenitors.

Because there is no surface antigen specific for endothelial progenitor cells (EPCs), it remains difficult to differentiate them from mature circulating endothelial cells. The CD34+, $\mathrm{vWF}+, \mathrm{CD} 31+$, vimentin + , c-kit- phenotype could represent either EPCs or mature endothelial cells. The absence of CD133 expression could indicate that these cells are not progenitor cells as defined by the expression of vascular endothelial growth factor receptor 2 (VEGFR2) and CD133. ${ }^{33}$ However, recently, our group has described the homing of endothelial cells of fetal origin in maternal inflamed skin during gestation in a mouse model. Fetal cells homed specifically to the inflamed skin. They could express markers such as CD31 and VEGFR2. Most importantly, they were able to form complete and functional blood vessels suggesting they were progenitors. ${ }^{22}$ These findings are in accordance with our present study demonstrating the transfer of fetal cells with an endothelial phenotype to the mother. In the absence of functional studies, however, it is difficult to ascertain the presence of a progenitor in the described fetal endothelial population.

An interesting application of these findings would be the isolation of fetal endothelial cells in maternal blood for prenatal diagnosis. Previous attempts using EPC culture systems or cell-surface markers such as CD34, CD133, CD105 and VEGFR2 in maternal blood during pregnancy have failed to isolate fetal cells in accordance with our results. ${ }^{34,35}$ More recently, fetal cells have been successfully isolated from the peripheral blood of pregnant women during the first trimester allowing male sex determination in $71 \%$ of tested samples. The isolated cells were among maternal adherent $\mathrm{CD} 34+$ cells and represented $0.5 \%$ of that population. These 
adherent $\mathrm{CD} 34+$ cells were most probably primitive mesenchymal progenitors because they expressed markers such as Oct-4 or Nanog usually present in embryonic stem cells. We believe our results are perfectly in line with the above-described findings. Even if the fetal CD34 + cells described here were obtained at term, they may have the adhesion properties previously described in circulating endothelial cells. ${ }^{36}$

In conclusion, we here showed that the IVS is a privileged site for the detection of the transfer of CD34 + fetal cells to the mother with an elevated cell concentration. These results also suggest that most $\mathrm{CD} 34+$ cells in maternal placental blood are of fetal origin. They are endothelial and not hematopoietic. Further studies are needed to assess the influence of such chimeric endothelial cells on maternal angiogenesis that may occur during inflammation, wound healing and tumor development.

Supplementary Information accompanies the paper on the Laboratory Investigation website (http://www.laboratoryinvestigation.org)

\section{ACKNOWLEDGEMENTS}

This work was supported by the Université Pierre et Marie Curie (BQR 2005 and 2007), the Fondation pour la Recherche Médicale (INE20050303543), the Association Française contre les Myopathies. We thank Marie-Josée Espié for technical assistance and Philippe Fontanges (IFR65, plate-forme imagerie Hôpital Tenon) for assistance in confocal microscopy. OP was supported by the Centre Hospitalo-Universitaire de Toulouse, Toulouse, France.

\section{DISCLOSURE/CONFLICT OF INTEREST}

None of the authors has any conflict of interest with the publication of this work.

1. Ariga $\mathrm{H}$, Ohto $\mathrm{H}$, Busch MP, et al. Kinetics of fetal cellular and cell-free DNA in the maternal circulation during and after pregnancy: implications for noninvasive prenatal diagnosis. Transfusion 2001:41:1524-1530.

2. Krabchi K, Gros-Louis F, Yan J, et al. Quantification of all fetal nucleated cells in maternal blood between the 18th and 22nd weeks of pregnancy using molecular cytogenetic techniques. Clin Genet 2001;60:145-150.

3. Holzgreve W, Ghezzi F, Di Naro E, et al. Disturbed feto-maternal cell traffic in preeclampsia. Obstet Gynecol 1998;9:669-672.

4. Bianchi DW, Farina A, Weber W, et al. Significant fetal-maternal hemorrhage after termination of pregnancy: implications for development of fetal cell microchimerism. Am J Obstet Gynecol 2001;184:703-706.

5. Khosrotehrani $\mathrm{K}$, Johnson $\mathrm{KL}$, Lau J, et al. The influence of fetal loss on the presence of fetal cell microchimerism: a systematic review. Arthritis Rheum 2003;48:3237-3241.

6. Bianchi DW, Williams JM, Sullivan LM, et al. PCR quantification of fetal cells in maternal blood in normal and aneuploid pregnancies. Am J Hum Genet 1997;61:822-829.

7. Nguyen Huu S, Dubernard G, Aractingi $S$, et al. Feto-maternal cell trafficking: a transfer of pregnancy associated progenitor cells. Stem Cell Rev 2006;2:111-116.

8. Bianchi DW, Zickwolf GK, Weil GJ, et al. Male fetal progenitor cells persist in maternal blood for as long as 27 years postpartum. Proc Natl Acad Sci USA 1996;93:705-708.

9. Khosrotehrani $\mathrm{K}$, Johnson $\mathrm{KL}, \mathrm{Cha} \mathrm{DH}$, et al. Transfer of fetal cells with multilineage potential to maternal tissue. JAMA 2004;292:75-80.

10. Srivatsa B, Srivatsa $S$, Johnson $K L$, et al. Microchimerism of presumed fetal origin in thyroid specimens from women: a case control study. Lancet 2001;358:2034-2038.
11. Wang $\mathrm{Y}$, Iwatani $\mathrm{H}$, Ito $\mathrm{T}$, et al. Fetal cells in mother rats contribute to the remodeling of liver and kidney after injury. Biochem Biophys Res Commun 2004;325:961-967.

12. Johnson $\mathrm{KL}$, Nelson JL, Furst $\mathrm{DE}$, et al. Fetal cell microchimerism in tissue from multiple sites in women with systemic sclerosis. Arthritis Rheum 2001;44:1848-1854.

13. Stevens AM, McDonnell WM, Mullarkey ME, et al. Liver biopsies from human females contain male hepatocytes in the absence of transplantation. Lab Invest 2004;84:1603-1609.

14. Tan XW, Liao $H$, Sun $L$, et al. Fetal microchimerism in the maternal mouse brain: a novel population of fetal progenitor or stem cells able to cross the blood-brain barrier? Stem Cells 2005;23:1443-1452.

15. Khosrotehrani K, Bianchi DW. Multi-lineage potential of fetal cells in maternal tissue: a legacy in reverse. J Cell Sci 2005;118:1559-1563.

16. O'Donoghue K, Chan J, De La FJ, et al. Microchimerism in female bone marrow and bone decades after fetal mesenchymal stem-cell trafficking in pregnancy. Lancet 2004;364:179-182.

17. Osada H, Doi S, Fukushima T, et al. Detection of fetal HPCs in maternal circulation after delivery. Transfusion 2001;41:499-503.

18. Guetta E, Gordon D, Simchen MJ, et al. Hematopoietic progenitor cells as targets for non-invasive prenatal diagnosis: detection of fetal CD34+ cells and assessment of post-delivery persistence in the maternal circulation. Blood Cells Mol Dis 2003;30:13-21.

19. Jimenez DF, Leapley AC, Lee Cl, et al. Fetal CD34+ cells in the maternal circulation and long-term microchimerism in rhesus monkeys (Macaca mulatta). Transplantation 2005;79:142-146.

20. Adams KM, Lambert NC, Heimfeld S, et al. Male DNA in female donor apheresis and CD34-enriched products. Blood 2003;102:3845-3847.

21. Khosrotehrani K, Leduc M, Bachy V, et al. Pregnancy allows the transfer and differentiation of fetal lymphoid progenitors into functional $T$ and B cells in mothers. J Immunol 2008;180:889-897.

22. Nguyen Huu $S$, Oster M, Uzan $S$, et al. Maternal neoangiogenesis during pregnancy partly derives from fetal endothelial progenitor cells. Proc Natl Acad Sci USA 2007;104:1871-1876.

23. Nguyen Huu S, Oster M, Avril MF, et al. Fetal microchimeric cells participate in tumour angiogenesis in melanomas occurring during pregnancy. Am J Pathol 2009;174:630-637.

24. Gekas C, Dieterlen-Lievre F, Orkin SH, et al. The placenta is a niche for hematopoietic stem cells. Dev Cell 2005;8:365-375.

25. Challier JC, Galtier M, Cortez A, et al. Immunocytological evidence for hematopoiesis in the early human placenta. Placenta 2005;26:282-288.

26. Johnson KL, Zhen DK, Bianchi DW. The use of fluorescence in situ hybridization (FISH) on paraffin-embedded tissue sections for the study of microchimerism. Biotechniques 2000;29:1220-1224.

27. Khosrotehrani K, Stroh H, Bianchi DW, et al. Combined FISH and immunolabeling on paraffin-embedded tissue sections for the study of microchimerism. Biotechniques 2003;34:242-244.

28. Khosrotehrani K, Guegan S, Fraitag S, et al. Presence of chimeric maternally derived keratinocytes in cutaneous inflammatory diseases of children: the example of Pityriasis lichenoides. J Invest Dermatol 2006;126:345-348.

29. Godin I, Cumano A. The hare and the tortoise: an embryonic haematopoietic race. Nat Rev Immunol 2002;2:593-604.

30. Bonney EA, Matzinger P. The maternal immune system's interaction with circulating fetal cells. J Immunol 1997;158:40-47.

31. Khosrotehrani K, Johnson KL, Guegan S, et al. Natural history of fetal cell microchimerism during and following murine pregnancy. J Reprod Immunol 2005;66:1-12.

32. Vernochet C, Caucheteux SM, Kanellopoulos-Langevin C. Bi-directional cell trafficking between mother and fetus in mouse placenta. Placenta 2007;28:639-649.

33. Urbich C, Dimmeler S. Endothelial progenitor cells: characterization and role in vascular biology. Circ Res 2004;95:343-353.

34. Gussin HA, Bischoff FZ, Hoffman R, et al. Endothelial precursor cells in the peripheral blood of pregnant women. J Soc Gynecol Investig 2002;9:357-361.

35. Gussin HA, Sharma AK, Elias S. Culture of endothelial cells isolated from maternal blood using anti-CD105 and CD133. Prenat Diagn 2004;24:189-193.

36. Mikhail MA, M'Hamdi $\mathrm{H}$, Welsh J, et al. High frequency of fetal cells within a primitive stem cell population in maternal blood. Hum Reprod 2008;23:928-933. 